\title{
MULTILATERATION UNDER FLIP AMBIGUITY FOR UAV POSITIONING USING ULTRAWIDE-BAND
}

\author{
Kunwoo Park ${ }^{1}$, Jungwon Kang ${ }^{1}$, Zahra Arjmandi ${ }^{1}$, Mozhdeh Shahbazi ${ }^{2}$, Gunho Sohn ${ }^{1 *}$ \\ ${ }^{1}$ Department of Earth and Space Science and Engineering, York University, 4700 Keele Street, Toronto, Ontario, M3J 1P3 Canada - \\ (kunwoo7, jkang99, zah.arjmandi, gsohn) @yorku.ca \\ ${ }^{2}$ Centre de géomatique du Québec, Saguenay, G7H 1 Z6 Canada - mshahbazi@cgq.qc.ca
}

Commission I, ICWG I/II

KEY WORDS: Multilateration, Flip Ambiguity, Positioning, Ultrawide-band, Unmanned Aerial Vehicle

\begin{abstract}
:
Ultrawide-band (UWB) ranging technology and multilateration techniques have recently been emerging solutions for positioning unmanned aerial vehicles (UAVs) in GNSS-denied environments. This solution offers cm-level ranging accuracy and considerable robustness to multipath receptions. UWB modules are commonly used in an anchor-based configuration; i.e., one UWB tag is mounted on the UAV, and several UWB anchors are installed on the ground. In real-world operational conditions, anchors can form a planar or a near-planar surface. This causes a geometric ambiguity, called flip ambiguity, in position estimation. Flip ambiguity can lead to considerable errors in the estimated position by multilateration. In this paper, we present a multilateration approach, which automatically resolves the flip ambiguity for UAV-positioning using UWB ranging. The proposed multilateration method first computes an algebraic solution through recursive least squares. If the initially estimated position is found to be flipped, then it is corrected by a symmetric reflection with respect to the anchor plane. Finally, the estimated position is refined by non-linear optimization. Extensive experiments in a real environment show that the proposed algorithm can effectively tackle the issue of flip ambiguity in multilateration, leading to a significant improvement in positioning accuracy.
\end{abstract}

\section{INTRODUCTION}

Recently, integrating three-dimensional vision to unmanned aerial vehicles (UAVs) has contributed a great deal to the advancement of geospatial technologies for fine-scale mapping, monitoring, and inspection applications. Examples are chimney inspection (Nieuwenhuisen et al., 2017), construction-site inspection (De Melo et al., 2017), and railway monitoring (Bertrand et al., 2017). Motivated by this growing potential of UAVs for inspection and monitoring applications, we have developed a UAV system with autonomous inspection capacity. The UAV system is named a quality-driven drone (Q-Drone). This drone is aimed to perform autonomous mapping of an unknown environment by generating its navigation path in a way that it can 1) maximize an objective function related to mapping quality, e.g. spatial resolution, 3D reconstruction accuracy, and modeling completeness; and 2) minimize the operation time by learning from human-designed flight operations.

In the context of autonomous mapping, the UAV system should be able to: start the operation and take off, navigate to a designated environment while generating its map, evaluate the quality of the generated map, and safely return to the home station (Bircher et al., 2016). In each task, the egocentric knowledge of the UAV in the environment is essential; that is, the vehicle should be able to estimate its position and orientation accurately, reliably, and robustly (Floreano and Wood, 2015). To this end, inertial navigation systems (INS) aided with GNSS, either in differential or standard mode, are the most commonly used technologies. However, in GNSS-denied environments (e.g., indoor, street canyons, and forested zones), these technologies fail to provide accurate position estimations due to multi-path receptions or loss of line-of-sight to the satellites (Zhu et al., 2018). In the absence of reliable GNSS observations, the accuracy of the INS decreases rapidly.

An emerging solution for positioning in GNSS-denied environments is ultra-wideband (UWB) ranging technology (Alarifi et al., 2016). The UWB transmission is a pulsed radiofrequency (RF) technology that transmits signals by generating radio energy at specific time intervals over a broad bandwidth with a very low power spectral density. Thanks to recent advancements in two-way-time-of-flight (TW-TOF) ranging techniques, we can achieve module-to-module range measurements at centimetre-level accuracy using UWB (Ruiz and Granja, 2017). With some UWB modules, long ranges can be measured up to a few hundred metres with an acquisition rate of up to $100 \mathrm{~Hz}$. Moreover, UWB ranging is known to be robust against multipath receptions and non-line-of-sight (NLOS) effects. Recent technology advancements allow us to access affordable pulsed-RF UWB modules (Johnson and Dewberry, 2011; Dewverry and Beeler, 2011; Dewberry and Petroff, 2013; Wang et al., 2010) for positioning UAVs and robots in both indoor and outdoor environments (Wang et al., 2017; Perez-Grau et al., 2017; Kanellakis, et al., 2019).

In this paper, UWB modules are used in an anchor-based configuration for positioning a UAV in GNSS-denied environments. Several UWB modules are installed as anchors at known positions on the ground, and one UWB module is attached as a tag to the UAV body (Figure 1). Through wireless communications in a round-robin manner, a series of range data between the tag and each anchor can be obtained. Therefore, the problem of UAV positioning can be formulated as estimating the location of the UWB tag relative to the anchors. To address this problem, multilateration techniques can be used. A large variety of multilateration approaches exist in the literature (Zhou et al.,

\footnotetext{
* Corresponding author
} 
2012; Norrdine, 2012; Li et al. 2017; Cotera et al., 2016). A significant factor dominating the performance of multilateration techniques is the geometric configuration of UWB anchors. For reducing the uncertainty in the estimated position, the anchors must not be co-planar or near co-planar. However, in real-world operational circumstances, this condition cannot always be satisfied. For instance, one can install the anchors using tripods of the same height located on flat terrain. Ill-configured anchors result in an ambiguity in the positioning solution, known as flip ambiguity (Liu et al., 2016). In other words, if the anchors are near co-planar, then multilateration results in two possible positions for the tag, one below the anchor plane and another above the anchor plane (Figure 2). There are several approaches for detecting flip ambiguity in 3D multilateration (Mautz et al., 2007; Liu et al., 2016), but none proposes a method for correcting a potentially flipped solution.

In this paper, we present a multilateration approach, which automatically resolves the flip ambiguity. The proposed multilateration method first computes an algebraic solution through recursive least squares. If the initially estimated position is found to be flipped, then it is corrected by being symmetrically reflected through the anchor plane. Finally, the estimated position is refined via non-linear optimization.

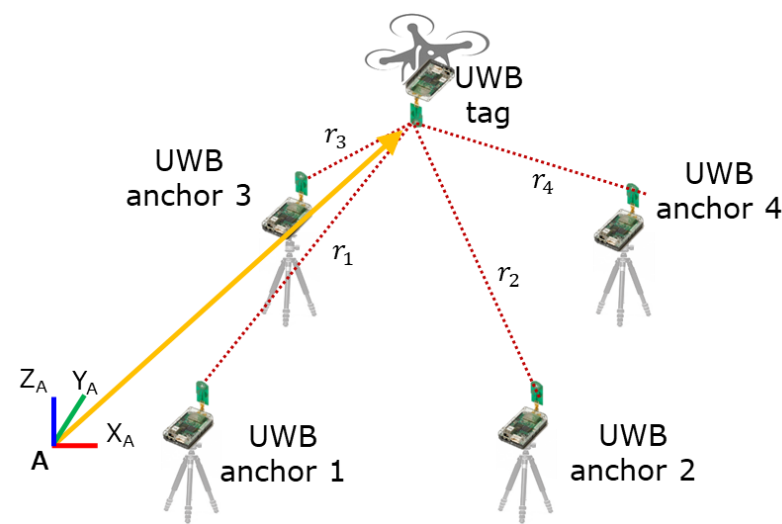

Figure 1. Description of UWB tag, UWB anchors and anchor frame

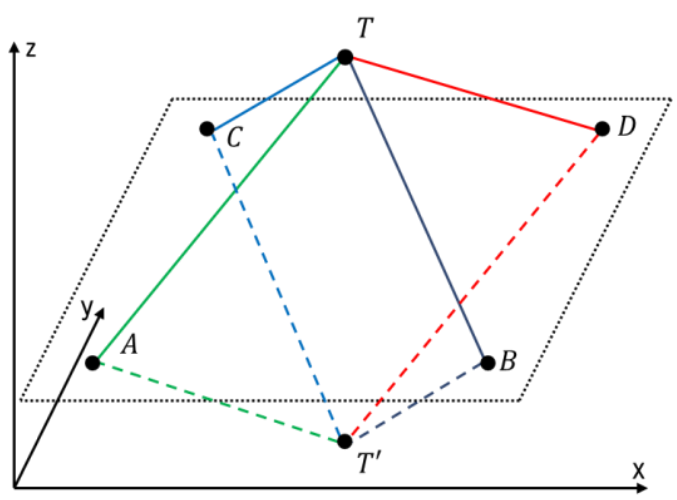

Figure 2. Flip ambiguity in 3D multilateration

\section{MULTILATERATION APPROACH}

In this section, we describe our method of multilateration under flip ambiguity. As shown in Figure 1, we suppose that one UWB module is attached to the UAV body, and a total of $n$ UWB anchors are installed on the ground, where $n \geq 3$. The anchor frame can be set on an arbitrary location. The position of each UWB anchor is assumed to be known in the anchor frame. The location of the $i$ th anchor in the anchor frame is denoted by $\mathbf{x}_{\mathrm{ua}}^{i}=\left[x_{\mathrm{ua}}^{i}, y_{\mathrm{ua}}^{i}, z_{\mathrm{ua}}^{i}\right]^{\mathrm{T}}$. Through sequential communications, each anchor transmits high-frequency range measurements to the base (a computer module on the ground). The range measurement received from anchor $i$ at time $t, r_{i, t}$, represents the distance between anchor $i$ and the UAV tag at that time. At any epoch, we collect a set $\boldsymbol{r}_{\boldsymbol{t}}=\left\{r_{1, t}, r_{2, t}, \cdots, r_{m, t}\right\}$ of range observations from $m$ different anchors, where $3 \leq m \leq n$.

The proposed multilateration approach consists of three significant steps (Figure 3). First, given a set of range observations $\boldsymbol{r}$ and the known position of the anchors, the position $\mathbf{x}_{\mathrm{s} 1}$ of the UWB tag is algebraically estimated. Next, $\mathbf{x}_{\mathrm{s} 1}$ is corrected if it is considered flipped. This correction is made by the symmetric reflection of $\mathbf{x}_{\mathrm{s} 1}$ with respect to the plane formed by the anchors (a.k.a anchor plane) resulting in $\mathbf{x}_{\mathrm{s} 2}$. In the last step, $\mathbf{x}_{\mathrm{s} 2}$ is refined to $\mathbf{x}_{\mathrm{s} 3}$ via non-linear optimization.

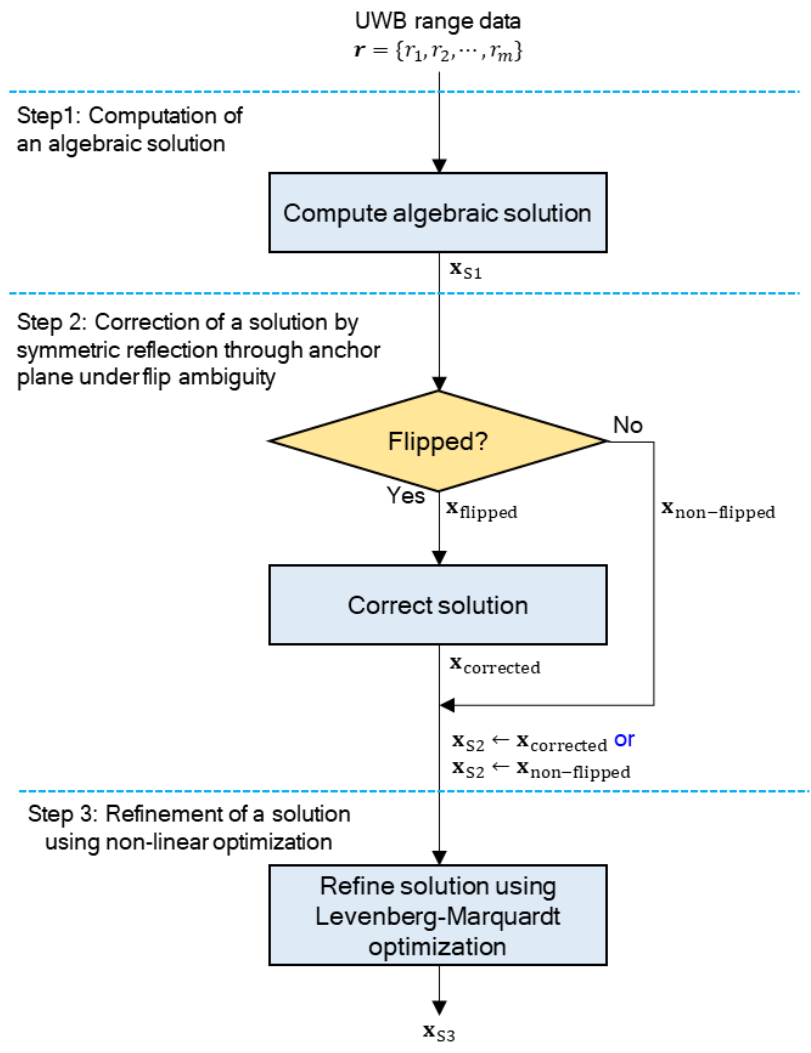

Figure 3. Proposed multilateration algorithm

\subsection{Algebraic Solution of the Tag Position}

Given a set of range observations, $\boldsymbol{r}=\left\{r_{1}, r_{2}, \cdots, r_{m}\right\}$ and the known position of the anchors in the anchor frame, we can estimate the approximate position of the tag $\mathbf{x}_{\mathrm{s} 1}=\left[x_{\mathrm{s} 1}, y_{\mathrm{s} 1}, z_{\mathrm{s} 1}\right]$ by solving the following system of equations. This solution follows the work of Norrdine (2012). Please note that the subscript $t$, denoting time, is dropped in the following equations for representation simplicity.

The nominal squared range between an anchor and the tag can be formulated as $\left\|\mathbf{x}_{\mathrm{s} 1}-\mathbf{x}_{\mathrm{ua}}^{i}\right\|_{2}^{2}$, which should be as close as possible 
to the observed $r_{i}^{2}$. This results in a system of equations $\mathrm{A}_{s 1} \mathbf{x}_{\mathrm{s} 1}^{\prime}=\mathbf{b}_{s 1}$, where

$$
\begin{aligned}
& \mathbf{x}_{\mathrm{s} 1}^{\prime}=\left[x_{\mathrm{s} 1}^{2}+y_{\mathrm{s} 1}^{2}+z_{\mathrm{s} 1}^{2}, x_{\mathrm{s} 1}, y_{\mathrm{s} 1}, z_{\mathrm{s} 1}\right]^{\mathrm{T}} \\
& \mathrm{A}_{s 1}=\left[\begin{array}{cccc}
1 & -2 x_{\text {ua }}^{1} & -2 y_{\text {ua }}^{1} & -2 z_{\text {ua }}^{1} \\
1 & -2 x_{\text {ua }}^{2} & -2 y_{\text {ua }}^{2} & -2 z_{\text {ua }}^{2} \\
\vdots & \vdots & \vdots & \vdots \\
1 & -2 x_{\text {ua }}^{m} & -2 y_{\text {ua }}^{m} & -2 z_{\text {ua }}^{m}
\end{array}\right]
\end{aligned}
$$

and

$$
\mathbf{b}_{s 1}=\left[\begin{array}{c}
r_{1}^{2}-\left(x_{\text {ua }}^{1}\right)^{2}-\left(y_{\text {ua }}^{1}\right)^{2}-\left(z_{\text {ua }}^{1}\right)^{2} \\
r_{2}^{2}-\left(x_{\text {ua }}^{2}\right)^{2}-\left(y_{\text {ua }}^{2}\right)^{2}-\left(z_{\text {ua }}^{2}\right)^{2} \\
\vdots \\
r_{m}^{2}-\left(x_{\text {ua }}^{m}\right)^{2}-\left(y_{\text {ua }}^{m}\right)^{2}-\left(z_{\text {ua }}^{m}\right)^{2}
\end{array}\right] .
$$

This system can be solved using recursive least squares.

\subsection{Resolving Flip Ambiguity}

In this work, we consider a ground-based anchor configuration, where each anchor is installed using a tripod on flat terrain. Since the height of the tripods does not vary much, anchors become near co-planar, and flip ambiguity occurs. In other words, whenever the following two configuration conditions are met, flight ambiguity is inevitable. 1) If the anchor-to-anchor distances are much larger than the height differences among the anchors. 2) If the anchors are located near the ground, and the flight altitude (tag-to-anchor distance) is much larger than the height differences among the anchors. These two conditions occur in many real-world operational circumstances.

To resolve the flip ambiguity, we assume that $\mathbf{x}_{\mathrm{s} 1}$ is flipped if $z_{\mathrm{s} 1}<T_{z}$, where we set $T_{z}=0$ indicating the ground level. By this rule, $\mathbf{x}_{\mathrm{s} 1}$ is classified into 'flipped' ( $\mathbf{x}_{\text {flipped }}$ ) or 'non-flipped' ( $\left.\mathbf{x}_{\text {non-flipped }}\right)$, as described in Figure $4 . \mathbf{x}_{\text {flipped }}$ is corrected to a new solution $\mathbf{x}_{\text {corrected }}$, while $\mathbf{x}_{\text {non-flipped }}$ is moved to the next step without further corrections.

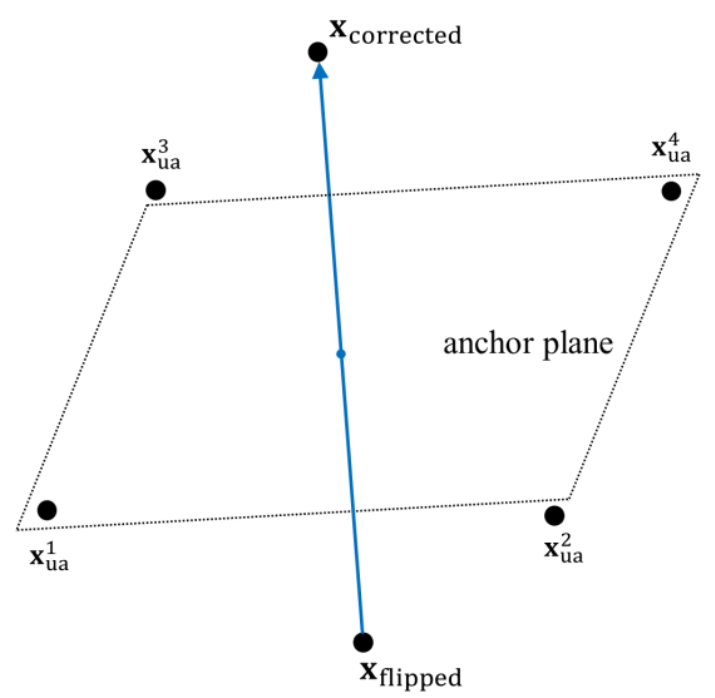

Figure 4. Symmetric reflection of a flipped solution through anchor plane

The correction is performed by symmetrically reflecting $\mathbf{x}_{\text {flipped }}$ with respect to the anchor plane. Figure 4 describes how $\mathbf{x}_{\text {flipped }}$ should be corrected to $\mathbf{x}_{\text {corrected }}$. First, assume that the model of the anchor plane is represented as $a x+b y+c z+1=0$. The plane parameters $\mathbf{p}_{\mathrm{ap}}=[\mathrm{a}, \mathrm{b}, \mathrm{c}]^{T}$ can simply be obtained by solving a linear system of equations $\mathrm{A}_{\mathrm{ap}} \mathbf{p}_{\mathrm{ap}}=\mathbf{b}_{\mathrm{ap}}$, where

$$
\mathrm{A}_{\text {ap }}=\left[\begin{array}{ccc}
x_{\text {ua }}^{1} & y_{\text {ua }}^{1} & z_{\text {ua }}^{1} \\
x_{\text {ua }}^{2} & y_{\text {ua }}^{2} & z_{\text {ua }}^{2} \\
\vdots & \vdots & \vdots \\
x_{\text {ua }}^{m} & y_{\text {ua }}^{m} & z_{\text {ua }}^{m}
\end{array}\right]
$$

and

$$
\mathbf{b}_{\mathrm{ap}}=-\mathbf{1}_{m \times 1} .
$$

Then, $\mathbf{x}_{\text {flipped }}$ is corrected to $\mathbf{x}_{\text {corrected }}$ by

$$
\mathbf{x}_{\text {corrected }}=\mathbf{x}_{\text {flipped }}-2 \cdot\left(\mathbf{p}_{\text {ap }} /\left\|\mathbf{p}_{\text {ap }}\right\|\right) \cdot \mathbf{v}
$$

where $\mathbf{v}=(\mathrm{a} x+\mathrm{b} y+\mathrm{cz}+1) /\left\|\mathbf{p}_{\mathrm{ap}}\right\|$. We denote $\mathbf{x}_{\text {corrected }}$ or $\mathbf{x}_{\text {non-flipped }}$ by $\mathbf{x}_{\mathrm{s} 2}$ as the outcome of this step.

\subsection{Non-linear Optimization}

The last step is to refine the solution using non-linear optimization. We refine $\mathbf{x}_{\mathrm{s} 2}$ to $\mathbf{x}_{\mathrm{s} 3}$ by solving

$$
\hat{\mathbf{x}}_{\mathrm{s} 3}=\arg \min _{\mathbf{x}_{\mathrm{s} 3}} \sum_{i} e_{i}^{2}
$$

where $e_{i}=r_{i}-\left\|\mathbf{x}_{\mathrm{s} 3}-\mathbf{x}_{\mathrm{ua}}^{i}\right\|_{2}$. Here, $\mathbf{x}_{\mathrm{s} 2}$ is used as an initial approximation of $\mathbf{x}_{\mathrm{S} 3}$ in the optimization. We used the Levenberg-Marquardt algorithm (Davis, 1993) for this optimization. As a result, we obtain $\mathbf{x}_{\mathrm{S} 3}$, which is the final output of our multilateration approach.

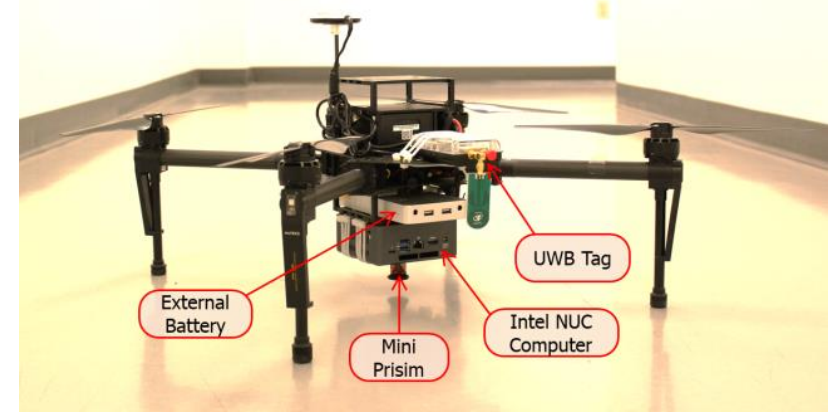

Figure 5. Q-drone with a UWB tag

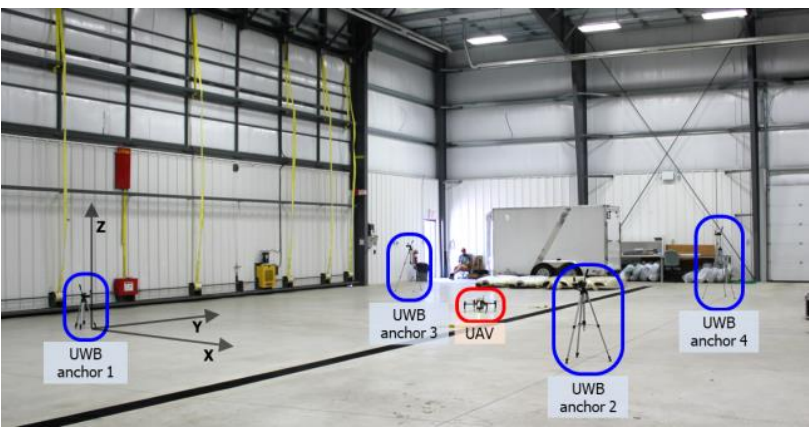

Figure 6. Experimental setup, showing the configuration of UWB anchors 


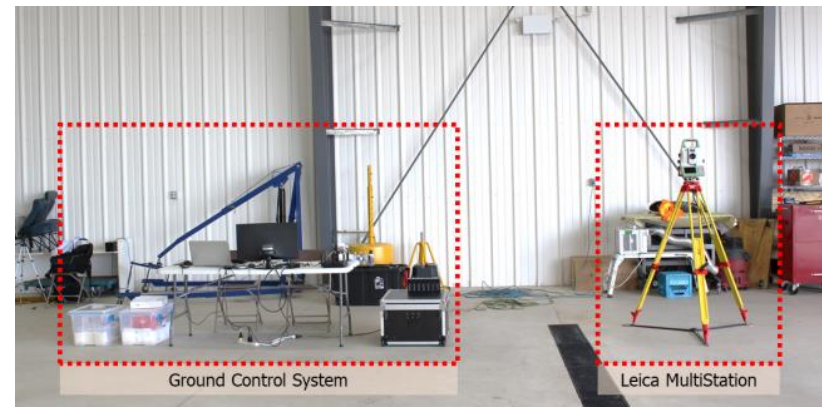

Figure 7. Ground control system and robotic total-station

\section{EXPERIMENTS AND RESULTS}

In this section, the experiments performed to assess the proposed UWB positioning approach are described, and the achieved results are discussed.

\subsection{UWB Modules and UAV System}

We used five TimeDomain P440 UWB modules for the experiments in this study. The TimeDomain P440 UWB modules provide range data with $2 \mathrm{~cm}$ accuracy from a maximum of 600 metres in clear-sighted conditions. One module was used as a tag attached to the UAV body (Figure 5), and the remaining four modules were used as anchors. We set the frequency to $10 \mathrm{~Hz}$ rate for communications between the tag and each anchor. Our UAV system was built on DJI M100, as shown in Figure 5. In addition to the UWB tag, an Intel NUC computer and one external battery were installed to acquire and store the data. Moreover, to obtain the ground-truth position of the UAV, a mini prism was attached underneath the UAV body.

\subsection{Experimental Setup and Acquired Datasets}

We conducted our experiments in an indoor space of $26 \mathrm{~m}$ (width) $\times 33 \mathrm{~m}$ (length) $\times 10 \mathrm{~m}$ (height), as shown in Figure 6 . The anchors $1,2,3$ and 4 were placed at coordinates $(0.00 \mathrm{~m}, 0.00 \mathrm{~m}$, $0.62 \mathrm{~m}),(8.51 \mathrm{~m}, 0.00 \mathrm{~m}, 0.96 \mathrm{~m}),(-0.26 \mathrm{~m}, 10.77 \mathrm{~m}, 1.32 \mathrm{~m})$ and $(8.25 \mathrm{~m}, 10.84 \mathrm{~m}, 1.58 \mathrm{~m})$, respectively. We used a robotic total-station, Leica Nova MS60 MultiStation, to measure the ground-truth position of the UAV. The MultiStation tracked the prism attached to the UAV and provided its position at a frequency of $10 \mathrm{~Hz}$ with mm-level of accuracy. The time of the MultiStation and the UAV were synchronized by comparing height measurements from the MultiStation and inertial measurement unit (IMU) integrated to the UAV.

\begin{tabular}{|c||r|r|r|r|r|}
\hline \hline & Set 1 & \multicolumn{1}{c|}{ Set 2 } & Set 3 & Set 4 & \multicolumn{1}{c|}{ Set 5 } \\
\hline \hline Duration (s) & 175.733 & 212.171 & 241.269 & 363.491 & 394.531 \\
\hline $\begin{array}{c}\text { Travelled } \\
\text { distance (m) }\end{array}$ & 138.831 & 167.494 & 210.485 & 298.466 & 448.229 \\
\hline x-min (m) & 2.592 & -0.281 & -0.474 & -0.440 & -1.403 \\
x-max (m) & 4.745 & 8.164 & 7.916 & 8.256 & 9.390 \\
\hline y-min (m) & 1.779 & -1.356 & -0.030 & 0.207 & -1.184 \\
y-max (m) & 12.689 & 13.082 & 9.970 & 8.474 & 10.174 \\
\hline z-max (m) & 6.910 & 6.087 & 7.164 & 6.212 & 5.347 \\
\hline \hline
\end{tabular}

Table 1. Description of the acquired datasets
In this experiment, we acquired five sets of data by manually navigating the UAV in different patterns. The specifications of the acquired datasets are summarized in Table 1. Each set of data includes range measurements taken by the anchors as well as the ground-truth position of the UAV.

\subsection{Results}

Figure 11 shows the errors in our positioning approach in each dataset. The mean absolute of these errors (MAE) are also summarized in Table 2. It can be concluded that our proposed approach for resolving/correcting flip ambiguity could improve the accuracy of UAV-positioning significantly. To clarify the impact of each processing step, the errors in the initial position, the flip correction, and the optimal position are summarized in Figure 8, Figure 9, and Figure 10.

\begin{tabular}{|c|c|c|c|c|c|c|c|}
\hline Solution & Position & Set 1 & Set 2 & Set 3 & Set 4 & Set 5 & Mean \\
\hline \multirow{4}{*}{$\mathbf{x}_{\mathrm{S} 1}$} & $\mathrm{x}$ & 0.081 & 0.076 & 0.076 & 0.055 & 0.089 & 0.075 \\
\hline & $\mathrm{y}$ & 0.121 & 0.113 & 0.097 & 0.084 & 0.110 & 0.105 \\
\hline & $\mathrm{z}$ & 1.463 & 1.314 & 1.250 & 0.915 & 1.364 & 1.261 \\
\hline & $\mathrm{xyz}$ & 1.494 & 1.343 & 1.273 & 0.938 & 1.396 & 1.289 \\
\hline \multirow{4}{*}{$\mathbf{x}_{\mathrm{s} 2}$} & $\mathrm{x}$ & 0.038 & 0.042 & 0.041 & 0.033 & 0.058 & 0.043 \\
\hline & $\mathrm{y}$ & 0.044 & 0.044 & 0.031 & 0.041 & 0.038 & 0.040 \\
\hline & $\mathrm{z}$ & 0.086 & 0.098 & 0.095 & 0.124 & 0.095 & 0.099 \\
\hline & xyz & 0.122 & 0.134 & 0.122 & 0.151 & 0.137 & 0.133 \\
\hline \multirow{4}{*}{$\mathbf{x}_{\mathrm{s} 3}$} & $\mathrm{x}$ & $\bar{~} 0.037$ & $\overline{0.041}$ & $\overline{\overline{0.040}}$ & $\overline{0.031}$ & $\bar{~} \overline{0.057}$ & 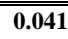 \\
\hline & $\mathrm{y}$ & 0.044 & 0.043 & 0.030 & 0.040 & 0.037 & 0.039 \\
\hline & $\mathrm{Z}$ & 0.079 & 0.089 & 0.084 & 0.118 & 0.087 & 0.091 \\
\hline & xyz & 0.114 & 0.125 & 0.112 & 0.145 & 0.129 & 0.125 \\
\hline
\end{tabular}

Table 2. Average positioning errors (Unit: metre)

\subsection{Discussion}

Here, we discuss the results obtained from each processing step, including (i) algebraic solution in step 1, (ii) correcting flip ambiguity in step 2, and (iii) non-linear optimization in step 3 .

3.4.1 Algebraic Solution: Figure 8 shows the errors in the initial solution $\mathbf{x}_{\mathrm{s} 1}$, which was estimated using the approach of Norrdine (2012). To clarify the errors caused directly by flip ambiguity, we separately present the errors in $\mathbf{x}_{\text {flipped }}$ and $\mathbf{x}_{\text {non-flipped }}$. As it can be seen, flip ambiguity caused gross positioning errors that could not be neglected. Positioning information with this level of inaccuracy could have severe consequences if used for navigating the UAV.

3.4.2 Flip Correction: Figure 9 shows the impact of the proposed method for resolving and correcting flip ambiguity (correcting $\mathbf{x}_{\text {flipped }}$ to $\mathbf{x}_{\text {corrected }}$ ). The errors caused by flip ambiguity could be significantly reduced by the proposed correction method.

3.4.3 Non-linear Optimization: Figure 10 shows the impact of positioning refinement by Levenberg-Marquardt non-linear optimization (refining $\mathbf{x}_{\mathrm{s} 2}$ to $\mathbf{x}_{\mathrm{s} 3}$ ). It can be noticed that nonlinear optimization slightly enhanced the accuracy of positioning. 


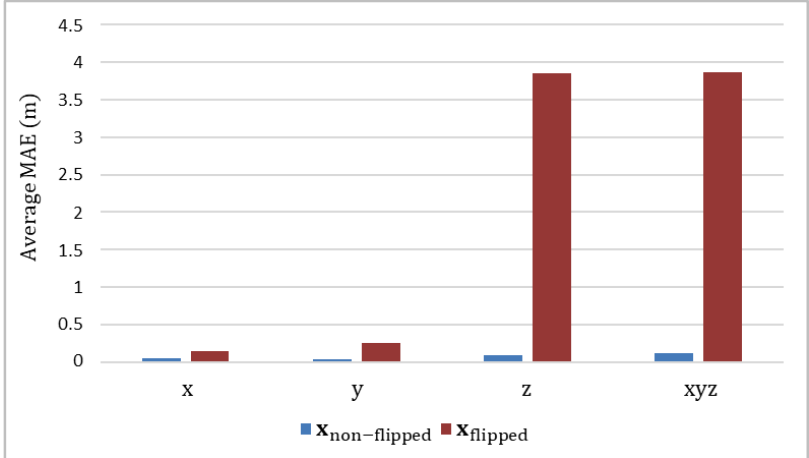

Figure 8. Average MAE for five data sets at Step 1.

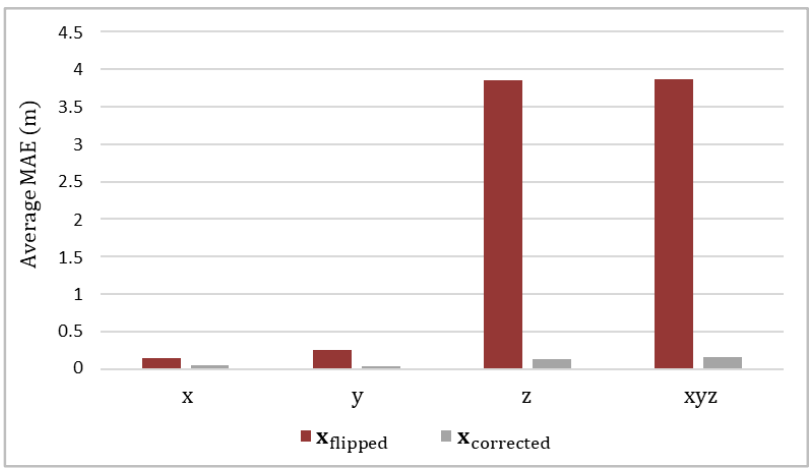

Figure 9. Average MAE for five data sets at Step 2.

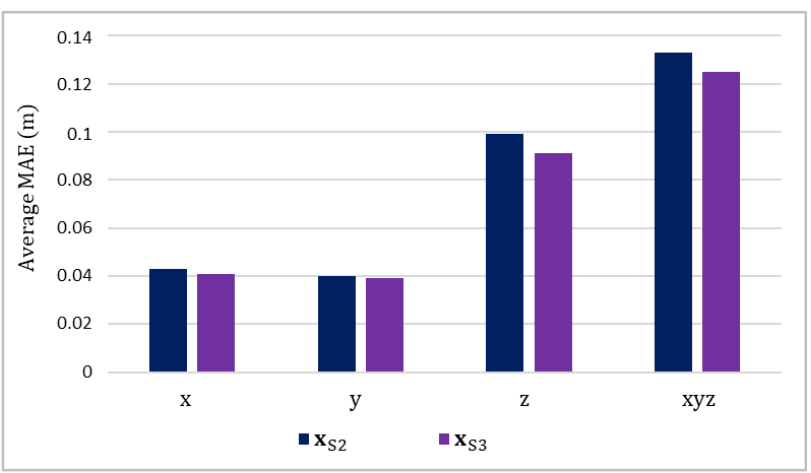

Figure 10. Average MAE for five data sets at Step 3.

\section{CONCLUSIONS}

In this paper, we presented a multilateration approach for positioning a UAV in GNSS-denied environments using the UWB ranging technology. Compared to the stet-of-the-art, the proposed multilateration approach benefits from an algorithm for resolving and correcting flip ambiguities. Through experiments in a real-world environment, we demonstrated that the proposed solution could effectively resolve the positioning errors caused by flip ambiguity. In general, an improvement of $89.68 \%$ in UAV-positioning accuracy was achieved by correcting the impact of flip ambiguities. Then, the results were further improved by $6.02 \%$ through non-linear optimization. In average, our complete UWB positioning workflow results in an average indoor positioning error of $0.125 \mathrm{~m}$.

\section{ACKNOWLEDGEMENT}

We would like to acknowledge the financial supports of this research from Transportation Canada's Rail Safety Improvement Program (RSIP), Mitacs Accelerate Program and the Natural
Science and Engineering Research Council of Canada (NSERC) Applied Research and Development (ARD) Program. Also, we would like to thank Centre de géomatique du Québec (CGQ) Inc. and Mr. Patrick Ménard at CGQ, who supported this research by providing surveying assistance to collect the reference data. We also want to express our gratitude to Teledyne Optech Inc. and Mr. Chris Verheggen, Senior Vice President of R\&D at Teledyne Optech Inc., who kindly allowed us to conduct our experiments in Teledyne Optech's indoor air cargo in Oshawa, Ontario. 

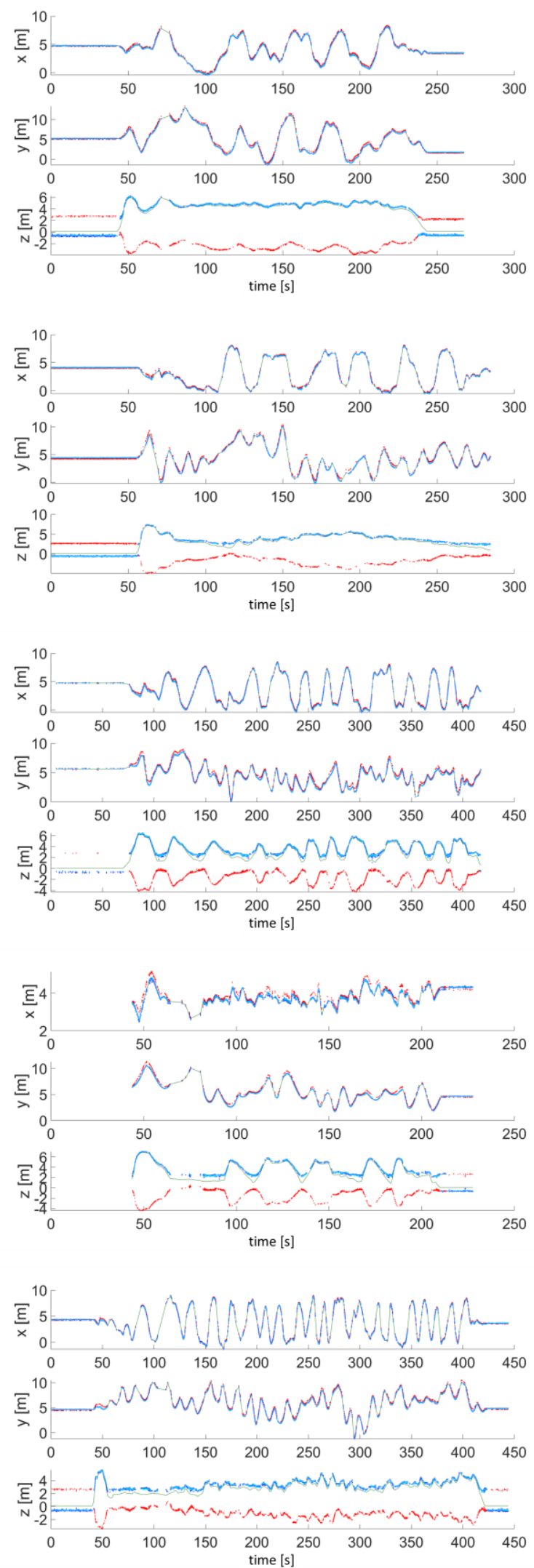

Set 4
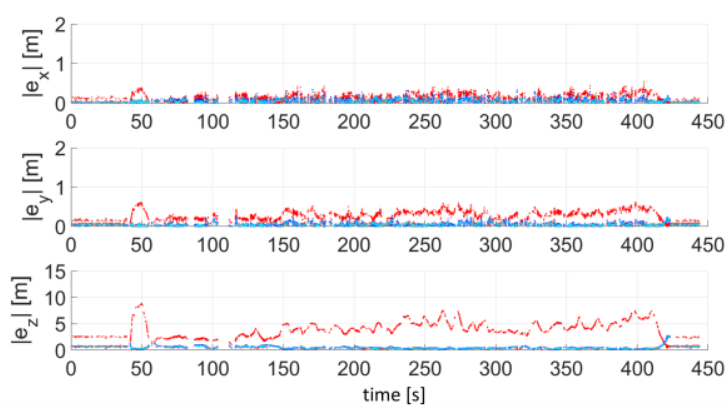

Set 5

Figure 11. Ground-truth trajectory and estimated position (left column) and MAE (right column) over time 


\section{REFERENCES}

Alarifi, A., Al-Salman, A., Alsaleh, M., Alnafessah, A., AlHadhrami, S., Al-Ammar, M. A., Al-Khalifa, H. S., 2016. Ultra wideband indoor positioning technologies: Analysis and recent advances. Sensors, 16(5), 707.

Bertrand, S., Raballand, N., Viguier, F., Muller, F., 2017, June. Ground risk assessment for long-range inspection missions of railways by UAVs. In 2017 International Conference on Unmanned Aircraft Systems (ICUAS), 1343-1351. IEEE.

Bircher, A., Kamel, M., Alexis, K., Oleynikova, H., Siegwart, R., 2016, May. Receding horizon" next-best-view" planner for 3d exploration. In 2016 IEEE international conference on robotics and automation (ICRA), 1462-1468. IEEE.

Cotera, P., Velazquez, M., Cruz, D., Medina, L., Bandala, M., 2016. Indoor robot positioning using an enhanced trilateration algorithm. International Journal of Advanced Robotic Systems, 13(3), 110.

Davis, P., 1993. Levenberg-marquart methods and nonlinear estimation. Siam News, 26(6), 1-12.

De Melo, R. R. S., Costa, D. B., Álvares, J. S., Irizarry, J., 2017. Applicability of unmanned aerial system (UAS) for safety inspection on construction sites. Safety science, 98, 174-185.

Dewberry, B., Beeler, B., 2011, June. Realtime range error estimation from pulse waveform signature analysis in support of extended kalman filter-based indoor navigation. In Proceedings of the Institute of Navigation (ION) 2011 Joint Navigation Conference, 26-28.

Dewberry, B., Petroff, A., 2013, October. SwarmNet: a distributed navigation network using ultra wideband ranging and communications. In 2013 European Microwave Conference, 338-341. IEEE.

Floreano, D., Wood, R. J., 2015. Science, technology and the future of small autonomous drones. Nature, 521(7553), 460-466.

Fornaser, A., Maule, L., Luchetti, A., Bosetti, P., De Cecco, M. (2019). Self-Weighted Multilateration for Indoor Positioning Systems. Sensors, 19(4), 872.

Johnson, J., Dewberry, B., 2011. Ultra-wideband aiding of GPS for quick deployment of anchors in a GPS-denied ad-hoc sensor tracking and communication system. Ion GNSS (Portland, OR Sep. 10-23, 2011), 1-8.

Kanellakis, C., Fresk, E., Mansouri, S. S., Kominiak, D., Nikolakopoulos, G., 2019. Autonomous visual inspection of large-scale infrastructures using aerial robots. arXiv preprint arXiv:1901.05510.

Li, J., Yue, X., Chen, J., Deng, F., 2017. A novel robust trilateration method applied to ultra-wide bandwidth location systems. Sensors, 17(4), 795.

Liu, W., Dong, E., Song, Y., 2016. Analysis of flip ambiguity for robust three-dimensional node localization in wireless sensor networks. Journal of Parallel and Distributed Computing, 97, 57-68.
Mautz, R., Ochieng, W., Brodin, G., Kemp, A. H., 2007. 3DWireless Network Localization from Inconsistent Distance Observations. Ad Hoc \& Sensor Wireless Networks, 3(2-3), 141170 .

Nieuwenhuisen, M., Quenzel, J., Beul, M., Droeschel, D., Houben, S., Behnke, S., 2017, June. ChimneySpector: Autonomous MAV-based indoor chimney inspection employing 3D laser localization and textured surface reconstruction. In 2017 International Conference on Unmanned Aircraft Systems (ICUAS), 278-285. IEEE.

Norrdine, A., 2012, November. An algebraic solution to the multilateration problem. In Proceedings of the 15th international conference on indoor positioning and indoor navigation, Sydney, Australia (Vol. 1315).

Onalaja, O., Adjrad, M., Ghavami, M., 2014. Ultra-widebandbased multilateration technique for indoor localisation. IET Communications, 8(10), 1800-1809.

Perez-Grau, F. J., Caballero, F., Merino, L., Viguria, A., 2017, September. Multi-modal mapping and localization of unmanned aerial robots based on ultra-wideband and RGB-D sensing. In 2017 IEEE/RSJ International Conference on Intelligent Robots and Systems (IROS), 3495-3502. IEEE.

Ruiz, A. R. J., Granja, F. S., 2017. Comparing ubisense, bespoon, and decawave uwb location systems: Indoor performance analysis. IEEE Transactions on instrumentation and Measurement, 66(8), 2106-2117.

Wang, C., Zhang, H., Nguyen, T. M., Xie, L., 2017, September. Ultra-wideband aided fast localization and mapping system. In 2017 IEEE/RSJ International Conference on Intelligent Robots and Systems (IROS), 1602-1609. IEEE.

Wang, D., Kannan, R., Wei, L., Tay, B., 2010, June. Time of flight based two way ranging for real time locating systems. In 2010 IEEE Conference on Robotics, Automation and Mechatronics, 199-205. IEEE.

Zhou, Y., Li, J., Lamont, L., 2012, December. Multilateration localization in the presence of anchor location uncertainties. In 2012 IEEE Global Communications Conference (GLOBECOM), 309-314. IEEE.

Zhu, N., Marais, J., Bétaille, D., Berbineau, M., 2018. GNSS position integrity in urban environments: A review of literature. IEEE Transactions on Intelligent Transportation Systems, 19(9), 2762-2778 